\title{
IDENTIDAD DE GÉNERO Y ACTITUDES SEXISTAS DEL ALUMNADO DE LA UNIVERSIDAD DE LAS PALMAS DE GRAN CANARIA
}

\author{
Míriam Lourdes Morales Santana \\ Universidad de Las Palmas de Gran Canaria miriam.morales@ulpgc.es \\ María Pilar Etopa Bitata \\ Universidad de Las Palmas de Gran Canaria pilar.etopa@ulpgc.es \\ Gabriel Díaz Jiménez \\ Universidad de Las Palmas de Gran Canaria gabriel.diaz@ulpgc.es
}

Recepción Artículo: 11 octubre 2021 Admisión Evaluación: 13 octubre 2021 Informe Evaluador 1: 14 octubre 2021 Informe Evaluador 2: 15 octubre 2021 Aprobación Publicación: 16 octubre 2021

\section{RESUMEN}

La internalización de actitudes sexistas posee una estrecha vinculación con la identidad de género en la medida en que influyen en nuestros pensamientos, emociones y comportamientos en contextos interpersonales e intergrupales. En la actualidad, la identidad de género no responde a una construcción dicotómica, al menos, aparentemente. Sin embargo, esto no implica necesariamente asumir que las actitudes sexistas hayan desaparecido; más bien se han transformado, adquiriendo un matiz más sutil y encubierto. Así, el análisis de la identidad de género y de la prevalencia de las actitudes discriminatorias basadas en el género constituyen el punto de partida para desarrollar intervenciones socioeducativas en materia de igualdad. La presente investigación analiza la identidad de género y las actitudes sexistas en una muestra de 1091 estudiantes universitarios de la Universidad de Las Palmas de Gran Canaria. El objetivo es examinar la prevalencia de actitudes discriminatorias y su relación con la identidad de género. Se exploran las relaciones entre las variables sometidas a estudio. En términos generales, los resultados muestran que la prevalencia de las actitudes sexistas oscilan entre niveles bajos y ambivalentes, con resultados similares en sexismo benévolo para ambos sexos y con una prevalencia superior de sexismo hostil en hombres, por un lado; y que altas identificaciones con el género masculino o femenino llevan aparejadas mayores niveles de sexismo hostil y benévolo respectivamente, por otro. Este estudio destaca la necesidad de incorporar medidas educativas que contribuyan a la igualdad de género en la enseñanza superior.

Palabras clave: género; identidad; actitudes sexistas; sexismo; prejuicio; igualdad

\section{ABSTRACT}

Gender identity and sexist attitudes of students from the University of Las Palmas de Gran Canaria. The internalization of sexist attitudes is closely linked to gender identity insofar as they influence our 
thoughts, emotions and behaviors in interpersonal and intergroup contexts. At present, gender identity does not respond to a dichotomous construction, at least apparently. However, this does not necessarily imply assuming that sexist attitudes have disappeared; rather they have been transformed, acquiring a more subtle and covert nuance. Thus, the analysis of gender identity and the prevalence of discriminatory attitudes based on gender constitute the starting point for developing socio-educational interventions on equality. This research analyzes gender identity and sexist attitudes in a sample of 1091 university students from the University of Las Palmas de Gran Canaria. The objective is to examine the prevalence of discriminatory attitudes and their relationship to gender identity. The significant differences in the variables under study and the predictive capacity of gender identity regarding sexist attitudes that hinder the change towards effective equality are explored. In general terms, the results show that the prevalence of sexist attitudes oscillate between low and ambivalent levels, with similar results in benevolent sexism for both sexes and with a higher prevalence of hostile sexism in men, on the one hand; and that high identifications with the male or female gender lead to higher levels of hostile and benevolent sexism respectively, on the other. This study highlights the need to incorporate educational measures that contribute to gender equality in higher education.

Keywords: gender; identity; sexist attitudes; sexism; prejudice; equality.

\section{INTRODUCCIÓN}

El género es, en palabras de García de León (2008), "uno de los pilares más vertebradores de todos los sistemas sociales" (p. 53). Esto es, las concepciones relativas al género condicionan aspectos individuales, grupales, estructurales y simbólicos. Así, se articula como un sistema de sistemas, un entramado extremadamente complejo que impregna la realidad social a todos los niveles.

Desde un enfoque psicosocial, masculinidad y feminidad como construcciones sociales se articulan como componentes fundamentales de la identidad social de género (Rocha y Díaz, 2015) en la medida en que señalan el lugar que los hombres y las mujeres ocupan dentro de la sociedad y condicionan a nivel individual la manera en que nos desenvolvemos en el sistema social (García de León, 2008a, b).

La identidad social, como la parte del autoconcepto, puede entenderse aquella dimensión en la que nos identificamos con determinadas categorías y nos definimos en base a ellas (Tajfel, 1981).

En este sentido, en tanto que los modelos de género constituyen creencias, son individualmente internalizados (Bourdieu, 2000) e influyen en nuestro comportamiento (Fiske, 1993, 1998; Gill, 2004; Heilman, 2001; Rudman y Glick, 2001; Prentice y Carranza, 2002) tanto a nivel interpersonal como intergrupal.

Por su parte, los estudios desarrollados en el ámbito de las actitudes intergrupales ponen de manifiesto el carácter sutil y ambivalente de los prejuicios en la actualidad (Briñol, Falces y Becerra, 2013; Glick y Fiske, 2011).

En su Teoría del Sexismo Ambivalente Glick y Fiske (1996) proporcionaron una explicación a las actitudes sexistas ambivalentes. En ella se definen dos tipos de actitudes hacia el género: el sexismo hostil y el sexismo benévolo. Por un lado, definen el sexismo hostil como una forma de manifestación tradicional de prejuicio, entendido como la actitud negativa y abiertamente manifestada hacia las personas en función de su sexo. Y por otro lado, el sexismo benévolo que consiste en la reformulación de las actitudes sexistas tradicionales, adquiriendo un matiz mucho más sutil y encubierto, sin dejar de responder a procesos de estereotipia. En conjunto, ambos tipos de sexismo contribuyen a la legitimación, justificación y mantenimiento de orden social respecto al género.

Además, el hecho de que la hostilidad no siempre se corresponda con la realidad informada y la sutileza con la que se expresan numerosas actitudes sexistas añade dificultad a la desarticulación de las desigualdades de género.

Actitudes que, aunque generalizadas en el plano social, estructural y simbólico, encuentran su nivel operativo básico en el ámbito individual; donde la robustez y prevalencia de los preceptos de género produce que las actitudes discriminatorias en función del sexo, como bien indica Bizer (2004), presenten un alto grado de estabilidad 
y poca variabilidad a lo largo del tiempo; una alta resistencia ante informaciones que la contradicen; y un gran número de efectos sobre el comportamiento en términos predictivos.

Con todo, la finalidad última de esta investigación radica en indagar sobre la prevalencia de modelos tradicionales de género y las actitudes discriminatorias que en consonancia se derivan de ellos en la población universitaria. Pretendemos examinar el contexto social de partida sobre el que construir de entornos pedagógicos con un alto contenido actitudinal que contribuyan a un desempeño personal, social y profesional, de acuerdo con valores de igualdad de trato y no discriminación.

\section{OBJETIVOS DE LA INVESTIGACIÓN}

El objetivo general de este estudio es identificar y analizar las relaciones existentes entre las variables identidad de género y actitudes sexistas. De manera concreta, pretendemos determinar, por un lado, la existencia de posibles diferencias en cada una de las variables incluidas en el estudio en función del sexo; y por otro, la relación existente entre las diferentes variables en función de la pertenencia a uno u otro sexo.

\section{MUESTRA Y/O PARTICIPANTES}

Participaron un total de 1091 estudiantes (706 mujeres y 385 hombres) de las titulaciones de grado impartidas en la Universidad de Las Palmas de Gran Canaria en la modalidad presencial, con una edad media de 22.1 años (DT= 5.03).

\section{METODOLOGÍA Y/O INSTRUMENTOS UTILIZADOS}

El presente estudio tiene un carácter descriptivo y correlacional. Se ha empleado un método de encuestación, utilizando una técnica de entrevista y administrando un cuestionario compuesto por un total de 28 ítems.

Se trata de la aplicación conjunta de la Escala de Detección de Sexismo en Adolescentes (DSA) (Recio, Cuadrado y Ramos, 2007) y dos ítems para medir la identidad de género.

Por una parte, la DSA esta compuesta por un total de 26 ítems, de los cuales 16 miden sexismo hostil y 10 sexismo benévolo. La escala de respuesta a todos los ítems oscila de 1 (totalmente en desacuerdo) a 6 (totalmente de acuerdo). La fiabilidad obtenida por los autores de la escala, medida mediante el Alfa de Cronbach, fue de .93 para el sexismo hostil y de .70 para el sexismo benévolo.

Por otra parte, López-Zafra y López-Saez (2001), demostraron mediante su investigación que la utilización de dos unicos ítems era suficiente para medir la identidad global de género. Se trata de ítems en los que se pregunta a los sujetos hasta qué punto se consideran femeninos/as y masculinos/as en una escala de 1 (nada) a 6 (mucho).

\section{RESULTADOS ALCANZADOS}

\section{Diferencias en las variables estudiadas en funcion del sexo}

En la Tabla 1 se presentan los estadísticos descriptivos, el coeficiente Alpha de Cronbach y el contraste de medias entre hombres y mujeres (estadístico de contraste: $t$ de Student) para el conjunto de variables estudiadas, con un nivel de confianza del $95 \%$. 
Tabla 1 Prueba t para la igualdad de muestras

\begin{tabular}{|c|c|c|c|c|c|c|}
\hline Variables & $N$ & $\begin{array}{c}\alpha d e \\
\text { Crombach }\end{array}$ & $M$ & $D T$ & $t$ & Sig \\
\hline \multicolumn{7}{|l|}{$\begin{array}{l}\text { IGG } \\
\text { masculina }\end{array}$} \\
\hline Mujer & 70 & -- & 2.2 & 1.2 & - & .00 \\
\hline Hombre & $\begin{array}{l}6 \\
38 \\
5\end{array}$ & & $\begin{array}{l}1 \\
5.1\end{array}$ & $\begin{array}{l}5 \\
.96\end{array}$ & $\begin{array}{c}39.4 \\
5\end{array}$ & 0 \\
\hline \multicolumn{7}{|l|}{$\begin{array}{l}\text { IGG } \\
\text { femenina }\end{array}$} \\
\hline Mujer & $\begin{array}{r}70 \\
6\end{array}$ & -- & $\begin{array}{c}5.5 \\
1\end{array}$ & .95 & $\begin{array}{c}45.9 \\
2\end{array}$ & $\begin{array}{l}.00 \\
0\end{array}$ \\
\hline Hombre & $\begin{array}{r}38 \\
5\end{array}$ & & $\begin{array}{c}2.1 \\
4\end{array}$ & $\begin{array}{l}1.1 \\
9\end{array}$ & & \\
\hline \multicolumn{7}{|l|}{ Sexismo } \\
\hline Mujer & $\begin{array}{r}70 \\
6\end{array}$ & .91 & $\begin{array}{c}1.2 \\
3\end{array}$ & .39 & $\begin{array}{c}- \\
7.15 \\
1\end{array}$ & $\begin{array}{l}.00 \\
0\end{array}$ \\
\hline Hombre & $\begin{array}{r}38 \\
5\end{array}$ & & $\begin{array}{c}1.5 \\
0\end{array}$ & .66 & & \\
\hline $\begin{array}{l}\text { Sexismo } \\
\text { Benévolo }\end{array}$ & & & & & & \\
\hline Mujer & $\begin{array}{r}70 \\
6\end{array}$ & .86 & $\begin{array}{c}2.1 \\
5\end{array}$ & .88 & $\begin{array}{c}- \\
1.90\end{array}$ & $\begin{array}{l}.05 \\
8\end{array}$ \\
\hline Hombre & $\begin{array}{r}38 \\
5\end{array}$ & & $\begin{array}{c}2.2 \\
6\end{array}$ & .87 & & \\
\hline
\end{tabular}

El análisis de la consistencia interna de los factores que componen la actitud sexista mediante el coeficiente Alpha de Cronbach ( ) arrojó una puntuación de .91 para sexismo hostil y de .86 para sexismo benevolente. La fiabilidad obtenida en los diversos factores es adecuada, ya que los valores son similares a los obtenidos en las muestras originales.

En el caso de los factores que constituyen la identidad de género no ha sido necesario realizar análisis de fiabilidad al tratarse de dos únicos ítems.

El contraste de medias en función del sexo para la variable identidad de género nos indica diferencias altamente significativas para ambos factores. En concreto, los hombres muestran puntuaciones superiores a las mujeres en identidad de género masculina, $t(1089)=-39.449, p<.001$, ocurriendo lo contrario en identidad de género femenina, $t(1089)=45.916, p<.001$.

Con respecto a las dos variables incluidas en el DSA, Ios resultados obtenidos muestran diferencias estadísticamente muy significativas en función del sexo para sexismo hostil $t(1089)=-8.228, p<.001$, donde los hombres $(M=1.50 ; D T=0.66)$ obtienen puntuaciones superiores a las mujeres $(M=1.26 ; D T=0.39)$. En sexismo benévolo no se hallaron diferencias significativas entre hombres y mujeres, $t(798)=-1.902, p=.058$.

Comparando ambos factores, podemos observar que las puntuaciones son superiores en sexismo benévolo en comparación con el hostil, aunque en ambos casos están por debajo del punto medio teórico de la escala. 


\section{Relacion entre las variables del estudio en hombres y mujeres}

Con objeto de conocer la relación entre las variables medidas en el estudio para la muestra global, en la Tabla 2 se presentan los resultados obtenidos mediante el cálculo del coeficiente de correlación de Pearson ( $r$ ) entre las variables estudiadas los coeficientes de correlación obtenidos para el total de la muestra.

Tabla 2 Correlación de Pearson entre variables para la muestra total

\begin{tabular}{lcccc}
\hline Medidas & 1 & 2 & 3 & 4 \\
\hline 1. IGG-M & - & & & \\
2. IGG-F & - & - & & \\
& $.81^{*}$ & & & \\
& $*$ & & & \\
3. SH & $.23^{*}$ & - & - & \\
& $*$ & $.24 *$ & & \\
& & $*$ & & \\
4. SB & .06 & -.04 & .59 & - \\
& & & $* *$ & \\
\hline $\begin{array}{l}\text { Nota. } \mathrm{N}=1091 .{ }^{*} p<.05 . \\
* * p<.01 .\end{array}$ & & & \\
\hline
\end{tabular}

Tal y como se puede apreciar, la identidad global de género masculina presenta una correlación negativa y significativa con identidad global de género femenina $(r=-.81, p<.01)$. Sin embargo, correlaciona positiva y significativamente con sexismo hostil $(r=$

.23, $p<.01)$.

Por su parte, la identidad global de género femenina muestra una correlación negativa con sexismo hostil $(r=-.23, p<.01)$.

Entre los factores que componen la DSA se ha obtenido una correlación positiva y estadísticamente significativa entre sexismo hostil y sexismo benévolo $(r=.59, p<.01)$. El análisis de correlación en función del sexo (Tabla 3), muestra que la identidad global de género masculina y femenina correlacionan negativa y significativamente, tanto para el grupo de hombres $(r=-.56, p<.01)$ como para el de mujeres $(r=-.50, p<.01)$.

Por su parte, el sexismo benévolo muestra correlación positiva y significativa con el sexismo hostil tanto para las mujeres $(r=.56, p<.01)$ como para los hombres $(r=.67, p<.01)$.

Asimismo, el sexismo hostil correlaciona positivamente con identidad de género masculina en el caso de los hombres $(r=.14, p<.01)$ y negativamente con identidad de género femenina $(r=-.11, p<.05)$. En el caso de las mujeres, el sexismo benévolo muestra una correlación positiva con la identidad de género femenina $(r=.07$, p<.05). 
Tabla 3 Correlación de Pearson entre variables medidas en función del sexo

\begin{tabular}{lcccc}
\hline Medidas & 1 & 2 & 3 & 4 \\
\hline 1. IGG-M & - & $-.50^{* *}$ & .2 & .00 \\
& & & 9 & \\
2. IGG-F & - & - & -.03 & .07 \\
& $.56^{*}$ & & & $*$ \\
& $*$ & & & \\
3. SH & $.14^{*}$ & $-.11^{*}$ & - & .56 \\
& $*$ & & & $* *$ \\
$4 . \mathrm{SB}$ & .06 & - & $.67 * *$ & - \\
& & .0 & & \\
& & 6 & & \\
\hline
\end{tabular}

Nota. Las correlaciones para el grupo de mujeres

$(n=706)$ se corresponden con los datos

ubicados por encima de la diagonal y las correlaciones para el grupo de hombres $(n$ $=385$ ) se corresponden con los datos ubicados por debajo de la diagonal. $* p<.05$. $* * p<$ .001 .

\section{DISCUSIÓN}

En términos generales, los resultados muestran diferencias significativas en función del sexo respecto a las variables identidad de género masculina, identidad de género femenina y sexismo hostil.

Con respecto a cuán masculinos/as y femeninos/as se sienten hombres y mujeres, se obtuvo que el sexo de Ios participantes está en correspondencia con la identidad de género tradicionalmente asociada al mismo. Esto es, las mujeres se identifican en mayor medida con la feminidad y en menor grado con la masculinidad; mientras que los hombres muestran resultados contrarios, declarándose más identificados con la masculinidad que con la feminidad. Por tanto, los resultados ponen de manifiesto que las dicotomías en torno a las que se construyen los modelos sociales de género tradicionales quedan reflejadas en la identidad global de género para la muestra analizada.

Asimismo, la relación inversa que se observa entre la identificación con el género masculino y femenino es similar en las investigaciones conducidas por López-Zafra y López-Sáez (2001) y Morales, Díaz y Etopa (2013). Resultados que indican que las categorías sociales de masculinidad y feminidad con las que puede identificarse una persona siguen operando de manera contrapuesta y con tendencia excluyente.

En relación al sexismo, aunque ambos factores se mantienen en puntuaciones bajas, se observa una mayor aceptación de manifestaciones benevolentes que hostiles. Tendencia que podría responder a cambios en las normas sociales y a la consecuente reformulación de las manifestaciones sexistas que contribuyen al mantenimiento y a la perpetuación de la desigualdad entre géneros (Brandt, 2011; Glick y Fiske, 2011; Glick et al., 2000; Ta demir y Sakallı-U urlu, 2010).

Basándose en diversos estudios, Dellacollette et al. (2012) entienden que la adopción de actitudes sexistas de carácter benévolo por parte y hacia las mujeres, suponen la aceptación de las prescripciones y proscripciones socialmente compartidas respecto al género, sobre todo en el contexto de las relaciones íntimas y no tanto en contextos laborales. De esta forma, se observa que el sexismo benévolo genera menos reacciones negativas en el ámbito privado (contexto comunal) y más en el ámbito laboral (contexto agéntico-instrumental).

Contrariamente a la deseabilidad social y los ideales igualitarios que se promulgan desde las sociedades occidentales, sí existen diferencias significativas en función del sexo para esta variable en el plano hostil. En con- 
sonancia con los resultados obtenidos por Morales, Díaz y Etopa (2013) y Esteban y Fernández (2017), Ios hombres presentan una tendencia a mostrar más actitudes sexistas hostiles hacia las mujeres que las propias mujeres.

Sin embargo, en el plano benévolo no se observan diferencias entre sexos. Estos resultados podrían confirmar carácter sutil y encubierto de este tipo de sexismo, lo que provoca que no sea percibido como una actitud negativa hacia las mujeres propiamente dicha y más socialmente aceptado, tal y como señalaron Glick y Fiske (1996).

Además, la relación directa que se produce entre las creencias sexistas hostiles y las benévolas, implica que cuanta más hostilidad se muestre hacia el género femenino, mayor es la probabilidad de mostrar actitudes sexistas sutiles y encubiertas. Resultado que evidencia la existencia de prejuicios ambivalentes hacia las mujeres que operan para perpetuar el status quo y legitimar la desigualdad estructural de género (Morales, 2015). Por su parte, las relaciones existentes entre la identidad de género y actitudes sexistas en función del sexo sugieren la influencia de patrones de género tradicionales que, aunque socialmente rechazados, siguen siendo comúnmente aceptados.

En el grupo de hombres, la identidad de genero masculina correlaciona positivamente con sexismo hostil mientras que la femenina muestra una relación inversa. Por otro

lado, la identidad de género femenina se relaciona de manera directa con el sexismo benévolo en el grupo de mujeres.

Además de corroborar el carácter ambivalente de las actitudes discriminatorias hacia las mujeres, tal y como formularon Glick y Fiske (1996, 1999, 2001a, 2001b), estos resultados revelan como la identificación con el modelo de feminidad - estereotipo valorado más negativamente a nivel social- se asocia al rechazo de manifestaciones hostiles y la aceptación de actitudes benévolas, aun siendo ambas discriminatorias, dada la necesidad de mantener un autoconcepto y una autoestima positivos.

La autocategorización en términos de maculinadad y/o feminidad requiere necesariamente de un proceso de identificación y, en efecto, deriva en un proceso de estereotipia. Así, las creencias, las normas y los valores asociados a una identidad social concreta (estereotipo), determinan en gran medida el comportamiento -incluidas las actitudes- de las personas que se identifican como miembros de dicha categoría (Herrera y Reicher, 2007; Bonilla, 2010)

Asimismo, el sexismo ambivalente no es cognitivamente conflictivo, puesto que reflejan actitudes complementarias que refuerzan ideologías tradicionales de género y contribuyen al mantenimiento del status quo. Mientras que el sexismo hostil, representa el castigo ante la resistencia de las mujeres a la dominación masculina; el sexismo benévolo, a modo de refuerzo, sirve de apoyo al mantenimiento de la división tradicional de género y, por consiguiente, a la interdependencia.

\section{CONCLUSIONES}

El género en su versión tradicional sigue siendo uno de los pilares más vertebradores de la sociedad a nivel individual y social. La correspondencia entre el sexo y la identidad de género sentida, así como la prevalencia de actitudes discriminatorias hacia las mujeres vinculadas, dejan constancia de la desigualdad, aún hoy día, existente entre hombres y mujeres.

De todos es sabido que el sexismo en su versión mas explícita no es una actitud aceptada socialmente. Sin embargo, el hecho de que sus manifestaciones abiertas tiendan a disiparse no es indicativo de que el prejuicio en sí haya desaparecido. Más bien, la sutileza se ha convertido en el tono expresivo de las actitudes sexistas.

Con todo, se evidencia la necesidad de ahondar en las manifestaciones sexistas actuales tras las que subyacen modelos tradicionales que obstaculizan la igualdad de género y, además, generar los mecanismos pedagógicos necesarios que promuevan actitudes socialmente favorables e igualitarias, así como la construcción/deconstrucción de la identidad basada en modelos estereotípicos de género tradicionales. 


\section{REFERENCIAS BIBLIOGRÁFICAS}

Bizer , G. (2004). Attitudes. En Encyclopedia of Applied Psychology (pp. 245- 249).

Elsevier. doi:10.1016/B0-12-657410-3/00559-6

Bonilla, A. (2010). Psicología y género: significación de las diferencias. Dossiers Feministes, 14, 129-150.

Bourdieu, P. (2000). La dominacion masculina. Barcelona: Anagrama.

Brandt, M.J. (2011). Sexism and Gender Inequality Across 57 Societies. Psychological Science, 22(11), 14131418. https://doi.org/10.1177\%2F0956797611420445

Brinol, P., Falces, C. y Berrera, A. (2013). Actitudes. En J. F. Morales Domínguez,

Psicologia social ( $3^{\mathrm{a}}$ ed.). McGraw-Hill.

Delacollette, N., Dumont, M., Sarlet, M. y Dardenne, B. (2013). Benevolent sexism, Men's advantages and the prescription of warmth to women. Sex Roles, 68(5), 296-310. doi:10.1007/s11199-012-0232-5

Esteban Ramiro, B. y Fernández Montaño, P. (2017). ¿Actitudes sexistas en jóvenes?: Exploración del sexismo ambivalente y neosexismo en población universitaria. FEMERIS: Revista Multidisciplinar de Estudios de Género, 2(2), 137-153. doi:http://dx.doi.org/10.20318/femeris.2017.3762

Fiske, S. T. (1993). Controlling other people. The impact of power on stereotyping.

American Pychology 48(6), 621-628.

García de León, M. A. (2008a). Eje de la violencia simbólica la masculinidad. CDC Cuadernos De Comunicacion, (2), 50-57.

García de León, M. A. (2008b), Rebeldes Ilustradas: La Otra Transición. Anthropos Editorial.

Gill, M. J. (2004). When information does not deter stereotyping: Prescriptive stereotyping can foster bias under conditions that deter descriptive stereotyping. Journal of Experimental Social Psychology, 40, 619-632. doi:10.1016/j.jesp.2003.12.001.

Glick, P. y Fiske, S. T. (1996). The Ambivalent Sexism Inventory: Differentiating hostile and benevolent sexism. Journal of Personality and Social Psychology, 70, 491-512.

Glick, P. y Fiske, S. T. (1997). Hostile and benevolent sexism: Measuring ambivalent sexist attitudes toward women. Psychology of Women Quarterly, 21(1), 119- 135. doi:10.1111/j.1471-6402.1997.tb00104.x

Glick, P., Lameiras, M., Fiske, S. T., Eckes, T., Masser, B., Volpato, C., Manganelli, A. M., Pek, J. C. X., Huang, L.-I., Sakalli-U urlu, N., Castro, Y. R., D'Avila Pereira, M. L., Willemsen, T. M., Brunner, A., Six-Materna, I., y Wells, R. (2004). Bad but Bold: Ambivalent Attitudes Toward Men Predict Gender Inequality in 16 Nations. Journal of Personality and Social Psychology, 86(5), 713-728. https://doi.org/10.1037/0022-3514.86.5.713

Glick, P., y Fiske, S. T. (1999). The ambivalence toward men inventory: Differentiating hostile and benevolent beliefs about men. Psychology of Women Quarterly, 23(3), 519-536. doi:10.1111/j.14716402.1999.tb00379.x

Glick, P., y Fiske, S. T. (2001a). Ambivalent sexism. En M. P. Zanna (Ed.), Advances in Experimental Social Psychology (pp. 115-188). Academic Press.

Glick, P., y Fiske, S. T. (2001b). Ambivalent stereotypes as legitimizing ideologies: Differentiating paternalistic and envious prejudice. En J. T. Jost y B. Major (Eds.), The psychology of legitimacy: Ideology, justice, and intergroup relations (pp. 278-306). Cambridge University Press.

Heilman, M. E. (2001). Description and prescription: How gender stereotypes prevent women's ascent up the organizational ladder. Journal of Social Issues, 57, 657-674. doi:10.1111/0022- 4537.00234.

Herrera, M. y Reicher, S. (2007). Categorización social y construcción de las categoría sociales. En J. F. Morales, M. Moya, E. Gaviria e I. Cuadrado (Coords), Psicologia social (3ạ ed., pp. 169-194). Mc Graw-Hill.

López-Zafra, E. y López-Sáez, M. (2001). Por qué las mujeres se consideran más o menos femeninas y los hombres más o menos masculinos: explicaciones sobre su autoconcepto e identidad de género. Revista de Psicología Social, 16(2), 193- 207.

Morales, M. (2015). Clima Social y Variables Psicosociales de Genero en la Universidad de Las Palmas de Gran 
Canaria [Tesis de doctorado no publicada]. Universidad de Las Palmas de Gran Canaria.

Morales, M., Díaz, G. y Etopa, P. (2013). Identidad de género y sexismo en estudiantes de segundo de bachillerato del norte de Gran Canaria. International Journal of Developmental and Educational Psychology, 1(1),451456.

Prentice, D. A. y Carranza, E. (2002). What women and men should be, shouldn't be, are allowed to be, and don't have to be: The contents of prescriptive gender stereotypes. Psychology of Women Quarterly, 26, 269-281. doi:10.1111/1471- 6402.t01-1-00066.

Recio, P., Cuadrado, I. y Ramos, E. (2007). Propiedades psicométricas de la Escala de Detección de Sexismo enAdolescentes (DSA). Psicothema, 19(3),522-528.

Rocha, T. y Díaz, R. (2011) Desarrollo de una escala para la evaluación multifactorial de la identidad de género en población mexicana. Revista de Psicologia Social: International Journal of Social Psychology, 26(2), 191206

Rudman, L. A. y Glick, P. (2001). Prescriptive gender stereotypes and backlash toward agentic women. Journal of Social Issues, 57, 743- 762. doi:10.1111/0022- 4537.00239.

Tajfel, H. (1981). Human groups and social categories. Cambridge University Press.

Ta demir, N. y Sakallı-U urlu, N. (2010). The relationships between am-bivalent sexism and religiosity among Turkish university students. Sex Roles, 62(7-8), 420-426. https://doi.org/10.1007/s11199-009-9693-6 
